New Concepts in Imaging: Optical and Statistical Models

D. Mary, C. Theys and C. Aime (eds)

EAS Publications Series, 59 (2013) 59-76

\title{
ASTRONOMICAL IMAGING... ATMOSPHERIC TURBULENCE? ADAPTIVE OPTICS!
}

\author{
M. Carbillet ${ }^{1}$
}

\begin{abstract}
This course/paper deals with adaptive optics in the framework of astronomical imaging. It does not pretend to be an exhaustive course of astronomical adaptive optics. It is rather intended to give an introductory overview of it, from my very partial point-of-view.
\end{abstract}

\section{Preamble: Images \& turbulence}

The image formed at the focus of ground-based telescopes is perturbed mainly by the last 10-20 km traveled by the light from the observed astronomical object, when propagating through the turbulent atmosphere. One has for the resulting image, and at the same time: scintillation, agitation, and spreading.

Scintillation is due to fluctuations of the global intensity of the image, this is the easily observed twinkling of stars. Agitation is the global variation of the photocenter of the formed image, which is due to tip and tilt of the incoming wavefront. Finally, spreading is due to the loss of spatial coherence of the incoming wavefront.

\subsection{Object-image relationship}

The object-image relationship which links the illumination $I(\boldsymbol{\alpha})$, in the focal plane of the telescope, where $\alpha$ is a bidimensional angular vector describing the line of sight, to the luminance $O(\boldsymbol{\alpha})$ of the object in the sky is a convolution implying the point-spread function (PSF) $S(\boldsymbol{\alpha})$ of the ensemble telescope $\oplus$ atmosphere:

$$
I(\boldsymbol{\alpha})=O(\boldsymbol{\alpha}) * S(\boldsymbol{\alpha}) .
$$

This relationship is valid notably at the condition that the system is invariant by translation, i.e. everything happens within the isoplanatic domain...

\footnotetext{
${ }^{1}$ UMR 7293 Lagrange, UNS/CNRS/OCA, Bât. Fizeau, Parc Valrose, 06100 Nice, France; e-mail: marcel.carbillet@unice.fr
} 

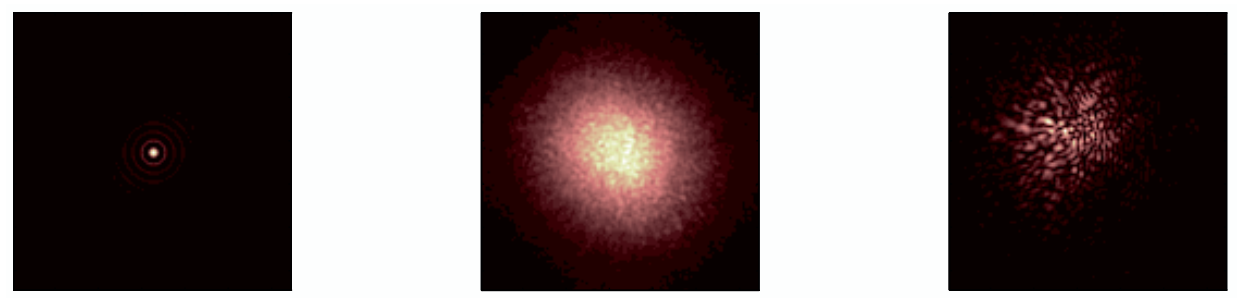

Fig. 1. Example of atmospherically-perturbed PSFs observed at the focus of a large ground-based telescope. From left to right: "ideal" Airy disc, long-exposure actuallyobserved PSF, and short-exposure actually-observed PSF. (From Carbillet 1996.)

Figure 1 shows an example of atmospherically-perturbed PSFs that can be observed at the focus of a ground-based telescope. The difference between the expected "ideal" Airy disc, the long-exposure actually-observed PSF (i.e. the image of an unresolved object), and the short-exposure actually-observed PSF (a speckle image) is dramatic. The Airy disc is showing a core of full-width at half-maximum (FWHM) $\lambda / D$, where $\lambda$ is the observing wavelength and $D$ the telescope diameter. The long-exposure actually-observed PSF is showing a core of FWHM $\lambda / r_{0}$, where $r_{0}$ is the typical size of the spatial coherence cells at the entrance of the telescope pupil (also called Fried parameter and detailed latter on - see next subsection). And the short-exposure actually-observed PSF is showing a speckle pattern which is changing very rapidly due to the time behavior of the turbulence.

\subsection{Some basic numbers}

Some basic numbers concerning the physical parameters driving the spatial and temporal behaviors of the atmospheric turbulence have to be remembered, in particular with respect to the observing wavelength $\lambda$.

Concerning spatial coherence, the basic factor over which everything is then built is the well-known Fried parameter $r_{0}$. This fundamental parameter directly gives the resulting angular resolution at the focal plane of the telescope: $\lambda / r_{0}$, quantity which is clearly independent of the telescope diameter $D$ (as far as $D$ is greater than $\left.r_{0}\right)$. In addition, $r_{0}$ being weakly dependent on the observing wavelength $\lambda$ (in fact $r_{0}$ is proportional to $\lambda^{6 / 5}$ ), this angular resolution (i.e. the FWHM of the resulting PSF) is roughly independent of $\lambda$ too. Writing down numbers, a typical $r_{0}$ of $10 \mathrm{~cm}$ in the visible (at $500 \mathrm{~nm}$ ) would correspond to $60 \mathrm{~cm}$ in the $\mathrm{K}$ band $(2.2 \mu \mathrm{m})$ and both would roughly correspond to a FWHM of the PSF of $\sim 1$ arcsec.

Concerning temporal coherence, the basic physical limitation comes this time from atmospheric turbulence layers velocity $v$, leading to an evolution time $\tau_{0} \simeq$ $r_{0} / v$. As it can be seen, $\tau_{0}$ is independent of $D$ but strongly dependent on $\lambda$. Typically $\tau_{0} \simeq 3 \mathrm{~ms}$ at a wavelength of $500 \mathrm{~nm}$ and $18 \mathrm{~ms}$ at $2.2 \mu \mathrm{m}$. 


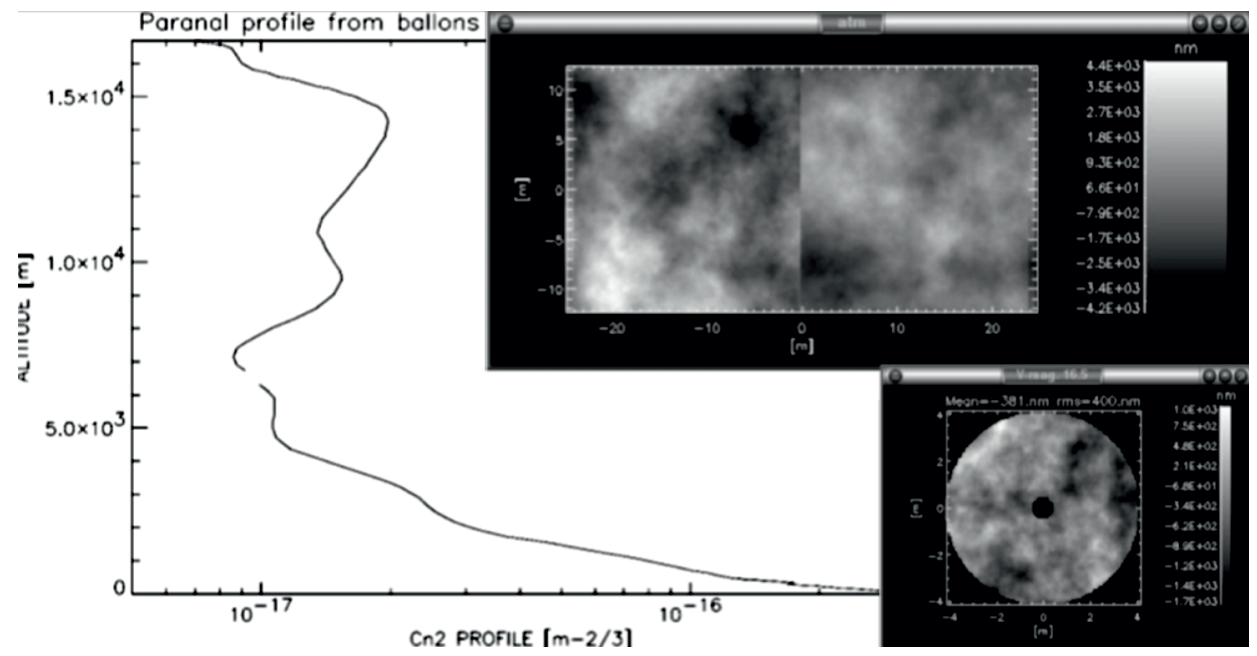

Fig. 2. Atmospheric turbulence. Left: typical atmospherical turbulent profile from a Mount Paranal site testing campaign (Sarrazin 1996). Top right: two $24 \mathrm{~m} \times 24 \mathrm{~m}$ modeled Kolmogorov/von Kármán turbulent layers, in terms of optical path difference. Bottom right: resulting wavefront propagated through these turbulent layers and to an 8-m telescope pupil. (From Carbillet 2006.)

Figure 2 shows a typical atmospherical turbulence profile, where layers are clearly identifiable, together with the representation of two modeled Kolmogorov/von Kármán turbulent layers and the resulting wavefront propagated through these turbulent layers and to the telescope pupil.

\subsection{Some basic equations}

The wavefront (measured in meters) is, by definition, proportional to the phase $\Phi(\boldsymbol{r})$ (measured in radians) by a factor $\frac{\lambda}{2 \pi}$. And $\Phi(\boldsymbol{r})$ is itself linked to the wave $\Psi(\boldsymbol{r})$, which traveled through the turbulent atmosphere, by the relation:

$$
\Psi(\boldsymbol{r})=A(\boldsymbol{r}) \exp \imath \Phi(\boldsymbol{r})
$$

where $A$ is the amplitude of the wave and $\boldsymbol{r}$ the bidimensional coordinate. Moreover, the phase $\Phi(\boldsymbol{r})$ can be decomposed on a polynomial basis, like for example the Zernike one, such as:

$$
\Phi(\boldsymbol{r})=\sum_{i} a_{i} Z_{i}(\boldsymbol{r})
$$

where $Z_{i}(\boldsymbol{r})$ represents the $i$-th Zernike polynomial and $a_{i}$ its related coefficient.

In addition to this general definition of wavefront and phase, one has to consider at least the principal equations which are ruling the atmospheric turbulence. The 
first one concerns the Fried parameter $r_{0}$, which is defined as (Roddier 1981):

$$
r_{0}=0.185 \lambda^{\frac{6}{5}} \cos \gamma^{\frac{3}{5}}\left[\int_{0}^{\infty} C_{n}^{2}(z) d z\right]^{-\frac{3}{5}},
$$

where $\gamma$ is the zenith angle and $C_{n}^{2}(z)$ is the structure constant of the fluctuations of the air refraction index $n$, which characterizes the optical energy of turbulence in function of the altitude $z$.

A number of typical parameters characterizing the resulting speckle pattern can be then deduced from it, such as the typical coherence time $\tau$, defined as (Roddier 1981):

$$
\tau_{0}=0.36 \frac{r_{0}}{v}
$$

or alternatively (Aime et al. 1986):

$$
\tau_{0}=0.47 \frac{r_{0}}{v}
$$

where $v$ is the mean velocity of the turbulent layers forming the turbulent atmosphere (weighted by the turbulence profile $C_{n}^{2}(z)$ ); but also the resulting "seeing":

$$
\epsilon=0.98 \frac{\lambda}{r_{0}}
$$

and the typical isoplanatic patch:

$$
\theta_{0}=0.36 \frac{r_{0}}{h}
$$

where $h$ is the mean height of the turbulent layers (weighted as well by the turbulence profile $\left.C_{n}^{2}(z)\right)$.

Finally, the wavefront perturbed by the turbulent atmosphere has a power spectral density which is classically modeled by (within the Kolmogorov/von Kármán model):

$$
\Phi_{\phi}(\boldsymbol{\nu})=0.0228 r_{0}^{-\frac{5}{3}}\left(\nu^{2}+\frac{1}{\mathcal{L}_{0}^{2}}\right)^{-\frac{11}{6}},
$$

where $\nu$ is the spatial frequency and $\mathcal{L}_{0}$ is the outer scale of turbulence (with a typical median value of $20-30 \mathrm{~m}$ for mid-latitude sites).

\subsection{The craftiness of speckle imaging and Lucky Imaging}

Before that the use of adaptive optics (AO) became a common thing for astronomy (since the first very convincing results of the mid-90's of last century), speckle imaging techniques were used in order to obtain high-angular resolution (HAR) images on large ground-based telescopes in the visible and near-infrared domains. A number of results were obtained, using first the pioneering visibility technique proposed by Labeyrie (Labeyrie 1970) and various others in the following - from 
the somehow raw shift-and-add technique (Worden et al. 1976) to more refined ones offered by bispectral imaging (Weigelt 1977), probability imaging (Aime 1987; Carbillet et al. 1998), cross-correlation (Aristidi et al. 1997) and others. The main idea under these techniques is that atmospheric perturbations can be frozen if the time exposure is less than $\tau_{0}$, and then some statistical invariant can be computed on a series of such short-exposure images in order to retrieve informations about the observed object.

Note that a selection of images can be done in order to select the best ones from a series of observations. Such observations were usually made of some thousands of images of a few milliseconds exposure, as many of the object that of an unresolved reference star, in order to obtain an estimate of the quantity which is computed also for the object images - e.g. spectrum, bispectrum, high-order probability density function, etc.. This idea is also basically the one under the Lucky Imaging (LI) technique (Baldwin et al. 2001) wich is commonly used since the advent of almost-readout-noise-free Electron-Multiplying CCD (EMCCD) detectors, and that is considered also for post-AO images (Mackay et al. 2012) for short (visible) wavelengths (were the AO correction is, at least for now, very partial).

\section{Adaptive optics}

The main problem of the previously described techniques is that the exposure time is limited, especially when considering classical CCD readout-noise-limited detectors, limiting hence sensitivity, signal-to-noise ratio, limiting magnitudes, and the like. Unlike AO, which in principle permits long exposure images (or spectra, or any other kind of data).

\subsection{Some basic numbers}

AO being designed to compensate atmospheric turbulence, the numbers evoked before (in terms of $r_{0}$ and $\tau_{0}$ ) are directly the first bricks of any AO instrument study. The typical size $d$ of each correcting element of a deformable mirror (DM) aimed to compensate the turbulence effects on the propagated wavefront, it hence follows that $d \simeq r_{0}$. As a consequence the total number of correcting elements becomes roughly $\left(D / r_{0}\right)^{2}$ which, with $D \simeq 10 \mathrm{~cm}$, is translated into approximately 7500 elements for a correction in the visible band, and 200 elements in $\mathrm{K}$ band. The same typical numbers are valid not only for correction (i.e. for the DM) but also, indeed, for what concerns the sensing of the incoming wavefront, through a given device (a wavefront sensor - WFS).

Temporal aspects are also very critical, since one would need to sample atmospheric turbulence at, let me say, a tenth of $\tau_{0}$. This leads to typical temporal frequencies for the whole $\mathrm{AO}$ system of $1 \mathrm{kHz}$ at $500 \mathrm{~nm}$ and $200 \mathrm{~Hz}$ at $2.2 \mu \mathrm{m}$.

Figure 3 schematizes the operation of a typical AO system: a perturbed wavefront enters the telescope, is reflected on a DM, sent to a beamsplitter dividing the light dedicated to the scientific device (a CCD, a spectrometer, whatever) and a WFS from which the collected information (e.g. spot centroids for computing 

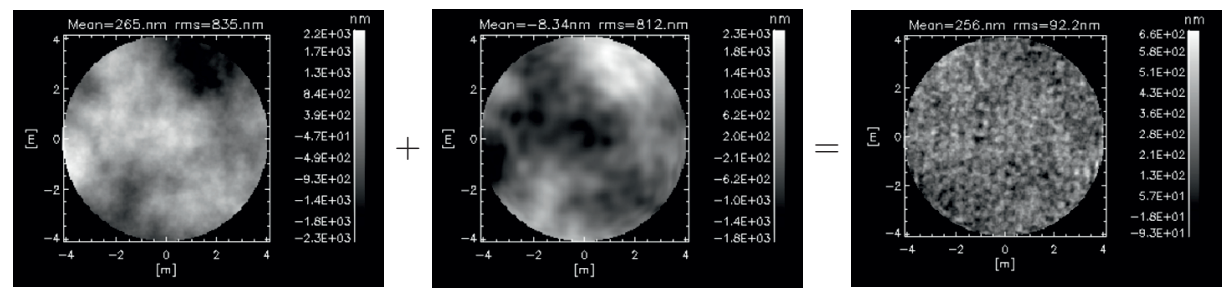

Fig. 3. AO system concept. Left: the incoming wavefront. Center: the DM shape, resulting from the commands sent from the wavefront reconstructor after analysis of the information collected from the WFS. Right: the resulting wavefront, after reflection of the input wavefront on the DM. (From Carbillet 2006.)

local wavefront slopes) is sent to a wavefront reconstructor that will elaborate DM commands from.

\subsection{The great variety of $A O$ "concepts" and their observational reasons}

We easily understand from the previous section that observing at HAR, at least with a monolithic telescope and not considering speckle techniques, needs an AO system, and that this AO system has to be dimensioned in function of the observing wavelength considered.

This is true but makes abstraction of a number of problems. The first of all is the number of photons necessary for wavefront analysis, or sensing. The analysis temporal frequency being necessarily very high (between $200 \mathrm{~Hz}$ and $1 \mathrm{kHz}$ in the illustration numbers given before), very bright stars are mandatory, dramatically reducing the portion of sky available for astrophysical observations (sky coverage).

One goal would then be to overcome this limitation in some way and have a $100 \%$ sky coverage. This is the goal of laser-guide-star (LGS) AO systems, which aim is to provide a sufficiently bright star in any direction of the sky, the closer possible to the observed object (Labeyrie \& Foy 1985). These artificial AO guide stars, are usually formed either from backscattering of the atmospheric sodium layer (situated at an altitude of $90-100 \mathrm{~km}$ ) or from Rayleigh scattering of the lower atmosphere (up to $\simeq 40 \mathrm{~km}$ ). This technique rises a number of additional problems, from the huge necessary power of the employed laser itself, to effects linked to the fact that the star is formed at a finite distance from the telescope, that it is clearly extended, and that tip-tilt is hardly sensed (the same tip-tilt being encountered in the upwards and subsequent downwards propagation).

A second problem which had been darkened till here is the problem of anisoplanatism, and hence very limited field of correction in which observing astrophysical objects around a suitable AO guide star. In order to limit this error and permit to decently observe faint objects a solution is to take advantage from a given number of possible surrounding guide stars nearby the interesting astrophysical object, hence considering multiple-reference AO systems. Such systems can be declined into at least three categories: multi-conjugate AO (MCAO) systems, ground-layer AO (GLAO) systems, and multiple-objects AO (MOAO) systems. 
MCAO systems aim at (partially) correct various layers of the turbulent atmosphere via DMs conjugated at different altitudes. At the opposite, GLAO is mono-conjugate and, in a simpler manner, aims at giving images (or spectra) corrected from the ground-layer turbulence only, since a great part of turbulence usually takes place within this layer. More peculiar, MOAO aims at correcting small fields in some directions of interest, within a much larger sensed field, through dedicated mirrors (one per direction of interest) and a global multiple-reference wavefront sensing.

Finally, the need to observe at very high-contrast levels in addition to HAR leads to the so-called "eXtreme" AO (XAO) systems, in which the basic concept is identical to a standard AO system, but each single component is pushed to its ultimate capacities and the whole system needs to break a number of conceptual and technological barriers.

\subsubsection{Importance of the observational goal}

A given class of astrophysical objects has its own observational priorities, such as the need to be directly detected and possibly spectrally characterized even at very low spectral resolution in the case of exoplanets, or for faint galaxies to obtain its precise morphology. As a consequence, this leads to consider the corresponding dominant AO errors (anisoplanatism in the faint-galaxies case, everything but anisoplanatism in the exoplanets case), and hence implies to consider ad hoc AO system concepts... for the present two examples: clearly MCAO, GLAO, or MOAO, possibly LGS-based, for the faint galaxies, and XAO for the exoplanets.

\subsection{The post-adaptive-optics error budget}

The post-AO error budget, in terms of variance integrated over the whole wavefront, is easily modeled by the following equation:

$$
\sigma_{\text {post-AO }}^{2}=\sigma_{\text {atmosphere }}^{2}+\sigma_{\text {AO system }}^{2}+\sigma_{\text {others }}^{2},
$$

where three basic quantities are present: the atmospheric error not considered by the AO system ( $\left.\sigma_{\text {atmosphere }}^{2}\right)$, the residual error from the AO system itself $\left(\sigma_{\mathrm{AO} \text { system }}^{2}\right)$, and finally other types of error neither due to the atmosphere nor to the AO system $\left(\sigma_{\text {others }}^{2}\right)$. Let me now have a detailed look into these three error terms.

\subsubsection{Errors not due to the (limited) adaptive optics correction}

Independently from the AO system considered, a number of errors, from both the physics of the (turbulent) atmosphere and the telescope/instrument are present. For what concerns the instrumental part the remaining error can be detailed as follows:

$$
\sigma_{\text {others }}^{2}=\sigma_{\text {calibration }}^{2}+\sigma_{\text {aberrations }}^{2}+\ldots
$$


where we see that this error is mainly coming from aberrations within the light path not seen by the AO system $\left(\sigma_{\text {aberrations }}^{2}\right)$, but also from possible calibration errors $\left(\sigma_{\text {calibration }}^{2}\right)$.

For what concerns the atmospheric effects, a number of them are not corrected at all by a standard AO system, as it is the case simply for scintillation, diffraction effects, chromatic effects, and indeed anisoplanatism, leading to:

$$
\sigma_{\text {atmosphere }}^{2}=\sigma_{\text {scintillation }}^{2}+\sigma_{\text {diffraction }}^{2}+\sigma_{\text {chromatism }}^{2}+\sigma_{\text {anisoplanatism }}^{2}
$$

Note that, in another hand, anisoplanatism is the main enemy when looking for wide-filed images, or simply faint objects far from a bright guide star.

\subsubsection{Errors due to the (limited) adaptive optics correction}

Within the AO-system error budget, a number of error sources can be identified, leading to the following formulation:

$$
\begin{aligned}
\sigma_{\text {AO system }}^{2} & =\sigma_{\text {fitting }}^{2}+\sigma_{\text {aliasing }}^{2}+\sigma_{\text {measure }}^{2}+\sigma_{\text {temporal }}^{2} \\
& +\sigma_{\text {LGS }}^{2}+\sigma_{\text {MCAO }}^{2}
\end{aligned}
$$

We will not detail here the last two terms which are strictly relevant to a LGS-based $\mathrm{AO}$ system $\left(\sigma_{\mathrm{LGS}}^{2}\right)$ and an MCAO system $\left(\sigma_{\mathrm{MCAO}}^{2}\right)$, respectively. Other specific errors can be defined if a specific AO system is considered.

Fitting Error. The first term of Equation (2.4) concerns the correction itself:

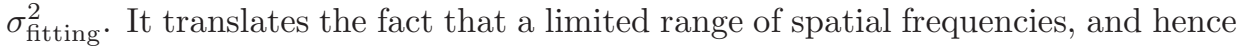
atmospheric turbulence modes, can be physically corrected by the mirror, and then the possible mirror modes. The reason is obvious and is simply linked to the total number of actuators building up the considered mirror. This error is consequently expressed in function of the ratio between the inter-actuators mean distance $d_{\mathrm{DM}}$ and the Fried parameter $r_{0}$ (in the imaging band considered):

$$
\sigma_{\text {fitting }}^{2} \propto\left(\frac{d_{\mathrm{DM}}}{r_{0}}\right)^{\frac{5}{3}}
$$

the exact coefficient of proportionality depending on the mirror construction itself and its ability to mimic atmospheric deformations.

Note that it is worthwhile to look at this error not only in terms of global average over the DM, but also in terms of spatial distribution, especially for segmented DMs. Figure 4 shows an example of the computed fitting error for the adaptive mirror M4 studied for the European Extremely Large Telescope (EELT), for median turbulence conditions (i.e., roughly speaking, the median value of $r_{0}$ ) for the EELT site. 


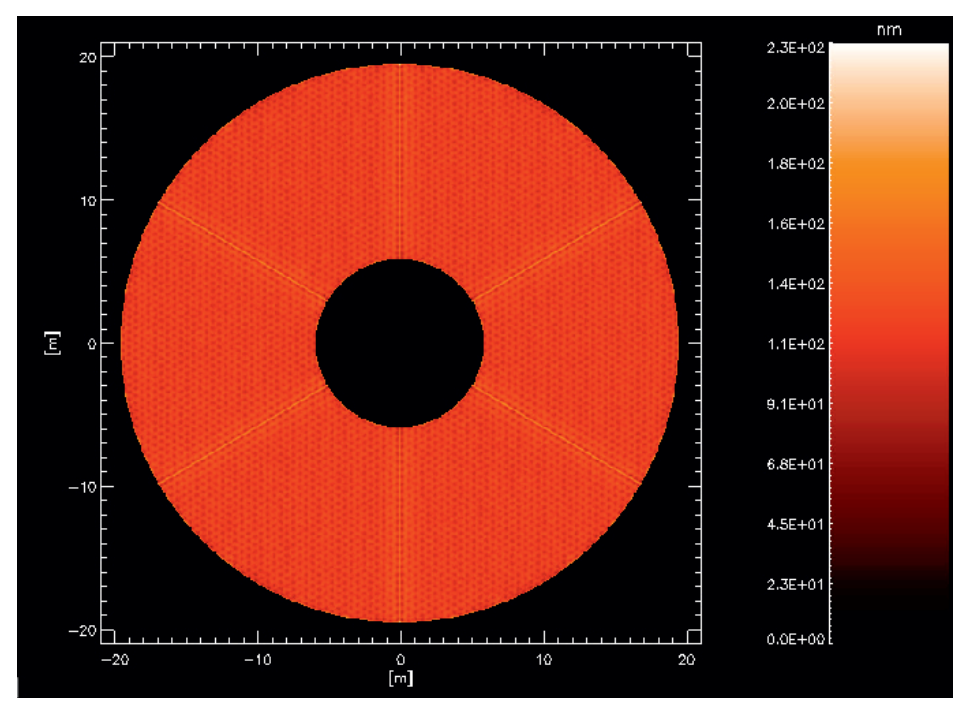

Fig. 4. Mean map of the residual rms wavefront, from which the fitting error can be deduced, for the adaptive mirror M4 of the EELT. (From Carbillet et al. 2012.)

Aliasing Error. The second error term of Equation (2.4) regards aliasing and is due to the WFS. Like the DM is limited by its finite number of actuators, the WFS is limited by its finite number of wavefront analysis elements (either the number of lenslets in the case of a Shack-Hartmann Sensor (SHS) or the number of pixels analyzing each of the 4 pupil images in the Pyramid Sensor (PS) case). Hence a problem of aliasing clearly appears because of the unseen spatial frequencies. Supposing that the physical size of the analysis elements of the WFS is $d_{\mathrm{WFS}}$, one has here also:

$$
\sigma_{\text {aliasing }}^{2} \propto\left(\frac{d_{\mathrm{WFS}}}{r_{0}}\right)^{\frac{5}{3}} .
$$

Let me note that very often $d_{\mathrm{WFS}} \simeq d_{\mathrm{DM}}$, but also that the geometry can still be completely different (e.g. circular for the DM and square for the WFS).

Measurement Error. The third term of Equation (2.4) is also related to the WFS, and more precisely to the measurement itself done by the WFS. This is a classical problem of light detection by a CCD device, where $\sigma_{\text {measure }}^{2}$ can be written:

$$
\sigma_{\text {measure }}^{2}=\sigma_{\text {photonization }}^{2}+\sigma_{\text {read-out }}^{2}+\sigma_{\text {dark-current }}^{2}+\ldots
$$

where the classical $\sigma_{\text {photonization }}^{2}$ error is clearly inversely proportional to the number of photons available $N_{\text {photons: }}$

$$
\sigma_{\text {photonization }}^{2} \propto\left(\frac{1}{N_{\text {photons }}}\right),
$$


and where the read-out noise (RON) error expresses in function of $N_{\text {photons }}$ and the associated variance $\sigma_{e}^{2}$ in terms of electrons/frame/second as:

$$
\sigma_{\text {read-out }}^{2} \propto\left(\frac{\sigma_{e}^{2}}{N_{\text {photons }}^{2}}\right) .
$$

Note that other minor errors such as the dark-current one can be considered too, and that almost-RON-free detectors such as EMCCDs (Electron-Multiplying CCDs) present in counterpart an "exotic" noise characterized by a Gamma distribution (instead of a Poisson distribution for the photon noise or a Gaussian one for the RON, see Carbillet \& Riccardi 2010).

Temporal Error. Last term evoked in Equation (2.4) is the one related to the global AO system temporal error, due to the simple fact that between the instant in which a given wavefront reflects on the DM and the instant in which it can be corrected by it (after measuring by the WFS, computing of the commands by the reconstructor and application of those commands by the DM), some milliseconds are usually gone. This error is indeed dependent on the turbulence coherence time $\tau_{0}$ and the total AO system "integration $\oplus$ delay" time $\Delta t_{\mathrm{AO}}$, and can be modeled as:

$$
\sigma_{\text {temporal }}^{2} \propto\left(\frac{\Delta t_{\mathrm{AO}}}{\tau_{0}}\right)^{\frac{5}{3}} .
$$

Balancing the Errors. It is clear from this list of errors that the main error sources for which a technological effort has to be done are, at least:

(1) $\sigma_{\text {fitting }}^{2}$ when designing the DM,

(2) $\sigma_{\text {aliasing }}^{2}$ and $\sigma_{\text {measure }}^{2}$ when choosing which WFS with which specific options has to be realized,

(3) and $\sigma_{\text {temporal }}^{2}$ when designing the whole $\mathrm{AO}$ loop.

Moreover, the critical physical parameters to be optimized are clearly:

(1) the inter-actuator distance (smaller and smaller),

(2) the number of analysis elements (higher and higher),

(3) the number of photons reaching the WFS (higher and higher),

(4) the global measurement variance (smaller and smaller),

(5) and the global "integration $\oplus$ delay" time (smaller and smaller),

where it is also straightforward that a number of trade-offs will have to be found. 


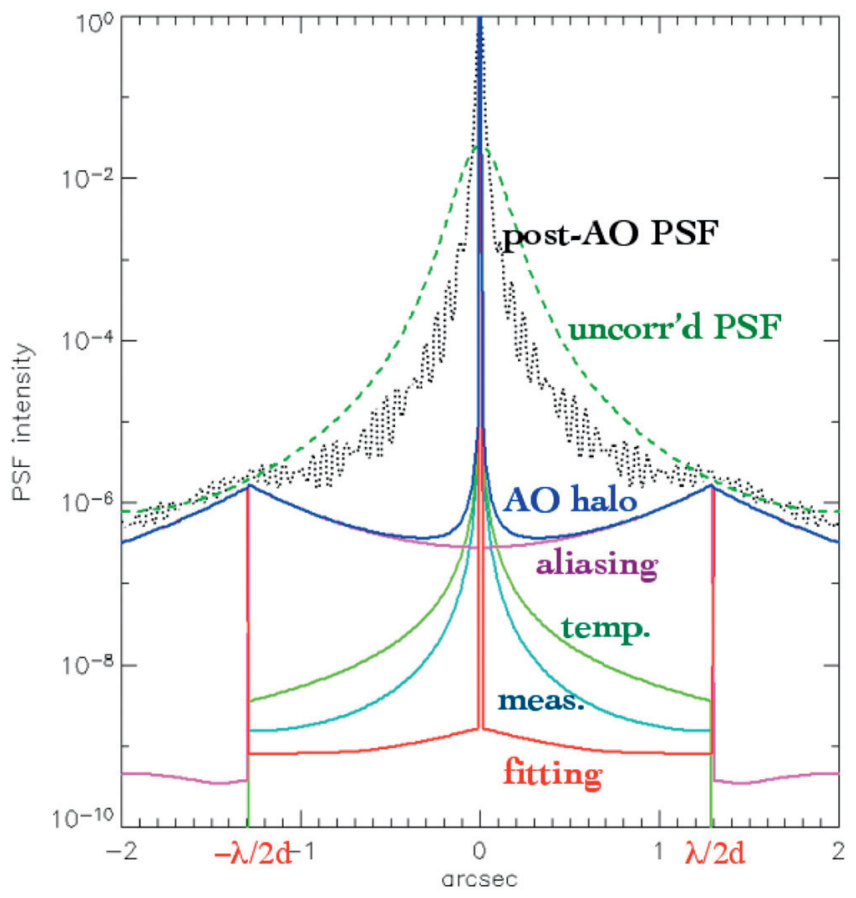

Fig. 5. Spatial/angular distribution of the different post-AO errors in the PSF halo. (From Lardière et al. 2005.)

\subsection{The (resulting) point-spread function morphology}

When separating the effects due to the different error sources from an AO system like I have done in the beginning of the present section, and more precisely looking at the spatial/angular distribution of these errors within the focal plane, i.e. within the PSF itself, we obtain what is represented in Figure 5.

The main interesting effect to observe from Figure 5 is the morphology of the fitting error and the aliasing error (here $d_{\mathrm{WFS}}=d_{\mathrm{DM}}=d$ ), especially around $\lambda / 2 d$, which gives this halo ring after which the Airy rings are not visible anymore and hence the benefit from AO correction is no more present. Like aliasing, the measurement error and the temporal error $\left(\sigma_{\text {measure }}^{2}\right.$ and $\sigma_{\text {temporal }}^{2}$ respectively) also participate for what concerns the angular resolution and to the distribution of error definitely inside the "cleared" $\lambda / d$ zone.

\subsection{Quality of correction?...}

The basic quantity permitting to characterize the AO-correction quality is indeed the Strehl ratio (Strehl 1902) (SR), which is defined as:

$$
S=\frac{I_{\text {post }-\mathrm{AO}}[0,0]}{I_{\text {perfect case }}[0,0]},
$$




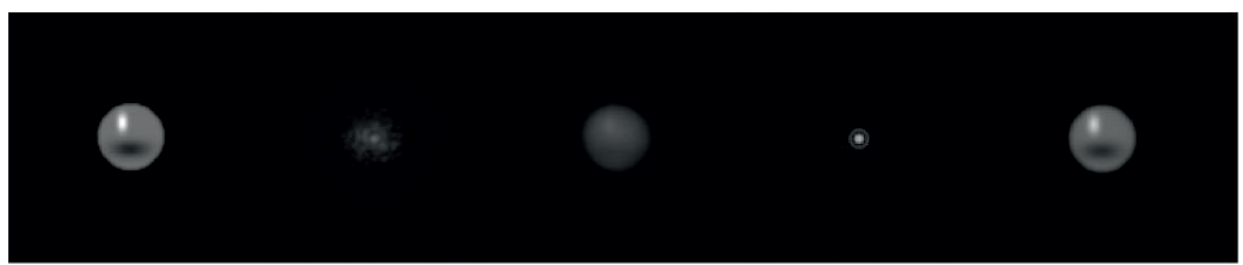

Fig. 6. From left to right: object, PSF with a SR of 0.07, resulting image, PSF with a SR of 0.93 , resulting image.

where $I_{\text {perfect case }}[0,0]$ is the intensity of the ideal PSF in its central point $([0,0])$ and $I_{\text {post-AO }}[0,0]$ corresponds to the same value but for the post-AO PSF.

Figure 6 shows the different effect that two different levels of attained SR have on the resulting HAR images: while the object is clearly recognizable in its various spatial details with a SR of 0.93 , it is almost unrecognizable with a SR of only 0.07 .

Nevertheless this could be far from being enough when a detailed study of the observational capabilities of a given instrument, with respect to a given observational goal, is necessary. In many cases alternative more descriptive quantities have to be used, such as:

(1) the attained FWHM of the PSF (when angular resolution is of main concern),

(2) the encircled energy for many spectrometric considerations,

(3) or for example the post-AO post-coronagraphic PSF wings level for very high-contrast questions.

Indeed all these alternative quantities are linked to the Strehl ratio obtained by a given system in a given observational situation, but not in an obvious linear manner.

More refined criteria for qualifying the AO correction can also be considered, especially when adapted to a given astrophysical goal. For example estimation of the attainable signal-to-noise ratio when dealing with detection problems (exoplanets, faint objects, etc.), or even the capability to obtain well-reconstructed images. In the latter one has typically to consider the whole imaging process: telescope $\oplus \mathrm{AO}$ system $\oplus$ instrument $\oplus$ data processing. Figure 7 details such an approach, where the capability for the whole imaging chain (starting here from the Large Binocular Telescope (LBT) in interferometric imaging mode) to obtain astrophysical informations on a given object is estimated through the precision obtained when reconstructing the magnitude difference between the components of the inner close binary star in one hand, and through the fidelity in retrieving the morphology of a very weak circumbinary ring in the other hand.

\subsection{The hard side}

An introduction on the concept and basic behavior of both the SHS and the PS can be found, e.g., in Campbell \& Greenaway (2006). I will focus here on the current duel that is featuring these sensors in particular in the framework of XAO. 

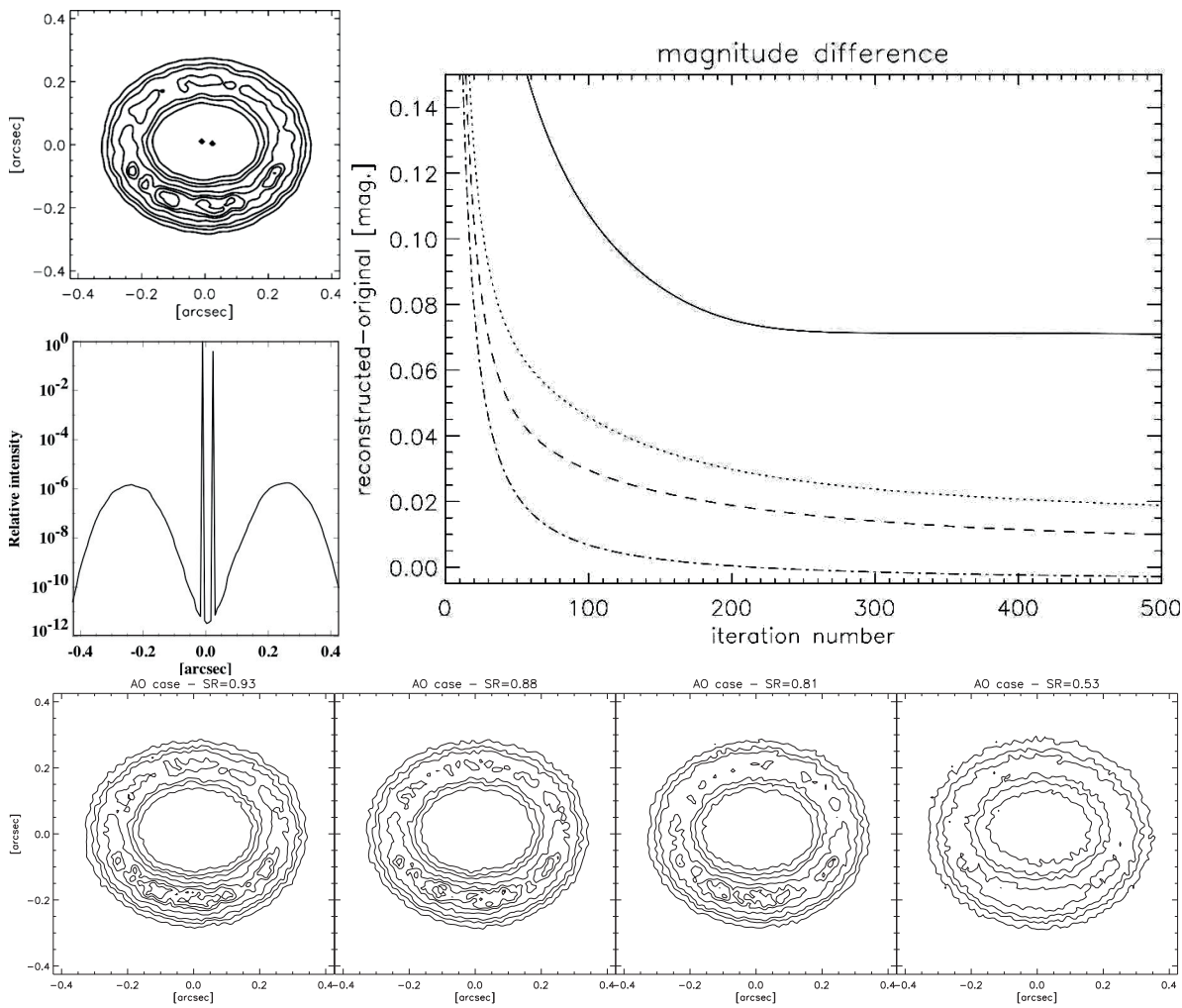

Fig. 7. Quality of post-AO K-band reconstructed images of a given object: a close double star surrounded by a circumbinary ring (more precisely a GG-Tau-like object). Top left: observed object (contour plot and cut along the inner binary star axis). Top right: quality of the reconstruction in terms of the reconstruction error on the magnitude difference between the components of the inner close binary system, for different Strehl ratios obtained. Bottom: quality of the reconstruction for the morphology of the circumbinary ring, for the same different Strehl ratios as before. (Adapted from Carbillet et al. 2002.)

Back in 1999 Ragazzoni \& Farinato (1999) shown, thanks to an analytic reasoning, that the PS should permit a gain of 2 magnitudes (in terms of limiting magnitude) with respect to its main competitor, the SHS. The analytic reasoning was based on the expression expected for $\sigma_{\text {measure }}^{2}$ for each Zernike component of the perturbed phase, expression derived from the result obtained previously by Rigaut \& Gendron (1992) for the SHS.

This was then confirmed by Esposito \& Riccardi (2001) by means of numerical simulations modeling AO correction (assuming weak phase perturbations), but in an open-loop regime and without any atmospherical residuals out of the modes corrected by the considered AO system.

Complete end-to-end simulations were then presented by Carbillet et al. (2003), considering this time the whole post-AO error $\sigma_{\text {post-OA }}^{2}$, and hence in particular 
$\sigma_{\text {measure }}^{2}$ in one hand and $\sigma_{\text {aliasing }}^{2}$ in the other hand. A gain was found in all the cases considered: in the photon-starving regime (where $\sigma_{\text {measure }}^{2}$ dominates) as, at the opposite, in the high-correction regime, where $\sigma_{\text {aliasing }}^{2}$ dominates.

The wind changed direction when Poyneer \& Machintosh (2004) proposed to diminish $\sigma_{\text {aliasing }}^{2}$ for the SHS by introducing a spatial filtering of each single spot behind the lenslet array. Moreover, Nicolle et al. (2004) proposed to diminish also $\sigma_{\text {measure }}^{2}$ by optimizing the signal calculations made after the SHS.

Finally Vérinaud et al. (2005) gave the definitive (but still theoretical) answer: while the PS better performs around the center of the diffraction pattern (i.e. around the core itself of the PSF), the (spatially filtered) SHS gives better results towards the edges of the previously evoked "cleared" $\lambda / d$ zone.

Since then, the PS has performed outstanding and unprecedented results on sky with FLAO, the first-light AO system of the LBT (see Esposito et al. 2010 \& Riccardi et al. 2010). The instrument SPHERE is expected to give similar results aboard the Very Large Telescope (VLT) from the SHS side... but it is still to be proven, at least for now, on sky.

\subsection{Deformable mirrors}

Different deformable mirrors technologies are being considered for the various AO systems existing or being developed world-wide: piezo-stacked mirrors, piezoelectric mirrors, MOEMS (Micro-Opto-Electro-Mechanical Systems - also called optical MEMS), adaptive secondary mirrors. They are all characterized at the very end by a few basic and fundamental parameters:

(1) the coefficient before the $d_{\mathrm{DM}} / r_{0}$ term in Equation (2.5),

(2) the inter-actuator distance $d_{\mathrm{DM}}$ itself,

(3) the mirror stroke,

(4) the response time necessary for a command to be executed by the mirror.

Concerning the first point listed before, it is completely linked to the morphology of the mirror itself when an actuator is pushed up, as clearly shown in Figure 8, where two different simple mirror technologies are shown to give two different mirror surface shapes and hence two different fitting error coefficients.

At this point a straightforward question has to be raised: how many actuators for a given achievable Strehl ratio? By only considering Equation (2.5) again, actual numbers to be given are (see Brusa et al. 1999):

$\star S_{\text {fit }} \simeq 0.75 \Rightarrow d \simeq r_{0}(\lambda)$ gives $N \simeq 350$,

$\star S_{\text {fit }} \simeq 0.92 \Rightarrow d \simeq 0.5 r_{0}(\lambda)$ gives $N \simeq 1450$,

considering band $\mathrm{J}$ and an 8-m class telescope.

The geometry (spatial distribution of the actuators) is another important point, the one that will determine the influence functions of the mirror, and hence its modes, the one that will be applied when a given command will be deduced by the wavefront reconstructor after each WFS measure. 


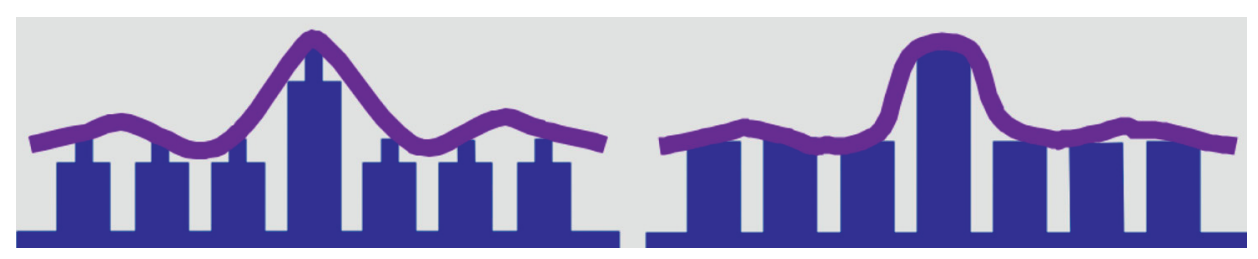

Fig. 8. Deformable mirror fitting illustration. (From Riccardi 2003.)

An interesting case to be further discussed is the adaptive secondary mirror one, for which a number of optical surfaces are eliminated, including the necessity of an additional tip-tilt mirror for usual DMs, leading to a considerable gain in number of photons available for WFS measures, and hence boosting the final performance of an AO system which uses such a type of DM (like it is used for FLAO/LBT, and will be used for the built-in M4 adaptive mirror of the EELT).

\subsection{Wavefront reconstruction and command control}

In order to have the DM applying the correct commands that will compensate the turbulent wavefront coming from the entrance pupil of the telescope, a wavefront reconstructor has to deduce the command needed to compensate the measured wavefront deformations (slope $x$ - and $y$-measurements from the WFS), and moreover: a command control has to be considered.

A very basic standard command makes use of a reconstruction process coupled with an integrator control law. The value of the gain of this integrator has to be optimized for a given AO system and a given guide star magnitude (and hence a given number of photons available per temporal unit), together with a number of AO system central parameters such as the WFS integration time and the number of DM modes to be corrected. A step forward consist in optimizing this gain mode by mode, as a function of the signal-to-noise ratio on each mode.

A (more refined) Kalman filter approach is also usually considered in order to command the system in an optimal way both for the reconstruction process and the control. The reader is invited to consult the course of J.-P. Folcher within these proceedings (Folcher 2013) for a detailed dissertation about this crucial subject.

\section{Going further}

\subsection{Various improvements are possible}

A number of improvements are definitely possible, as long as any term of $\sigma_{\text {post-AO }}^{2}$ can be diminished in some way. At least the three following possible improvements are currently investigated and seriously considered for AO systems:

(1) reduction of $\sigma_{\text {measure }}^{2}$ first: by using EMCCDs for the WFS. These devices have the capability to mimic a very low read-out noise. The counter part of it is nevertheless the addition of an "exotic" dark-current component. 

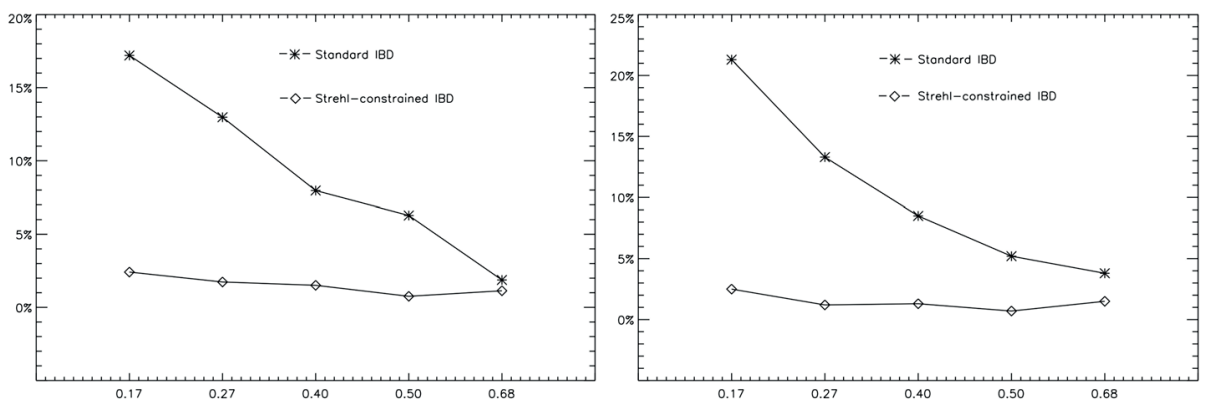

Fig. 9. Final error on the reconstruction of the PSF (left) and on the object (right), when using an IBD) algorithm. Both plots are made as a function of the SR of the image data and comparing the simple IBD (rhombuses) to the Strehl-constrained IBD (asterisks). A gain of up to a factor $\sim 10$ is achieved for the poorer SR. (From Desiderà \& Carbillet 2009.)

(2) reduction of $\sigma_{\text {measure }}^{2}$ second: by adding a dedicated tip-tilt sensor in addition to the global WFS. Then one has to find an optimal value for the splitting of light between the tip-tilt sensor and the higher-order WFS, and that this splitting of light is still of any advantage with respect to using a single WFS. The answer is not unique but depend on the precise AO system used - and in particular the WFS used (see, e.g., Carbillet et al. 2005): PS, SHS, filtered SHS, etc.

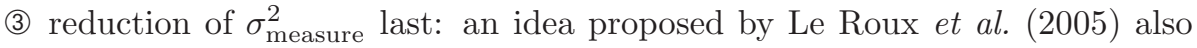
consider to mask the WFS - toward a coronagraphic WFS?

(4) reduction of $\sigma_{\text {atmosphere }}^{2}$ : this last error could be the most simple (from the conceptual point-of-view) but the most complicate (from the practical pointof-view) to diminish, since it could imply to consider to install the AOequipped telescope on a tower in the middle of Antarctica, since it can be seen as the best site on earth when eliminating the very thin turbulence surface layer (see, e.g., Lardière et al. 2005; Aristidi et al. 2009; Carbillet et al. 2010; Giordano et al. 2012).

\subsection{Post-adaptive-optics object reconstruction}

\subsubsection{Knowledge of the Quality of Correction $\Rightarrow$ Even Better Object Reconstruction}

Figure 9 shows the advantage of using a constraint on the Strehl ratio when reconstructing the PSF (in the cases where it is unknown or badly known), and hence the object from the obtained image, in the case of an iterative blind deconvolution (IBD) of post-AO data. 

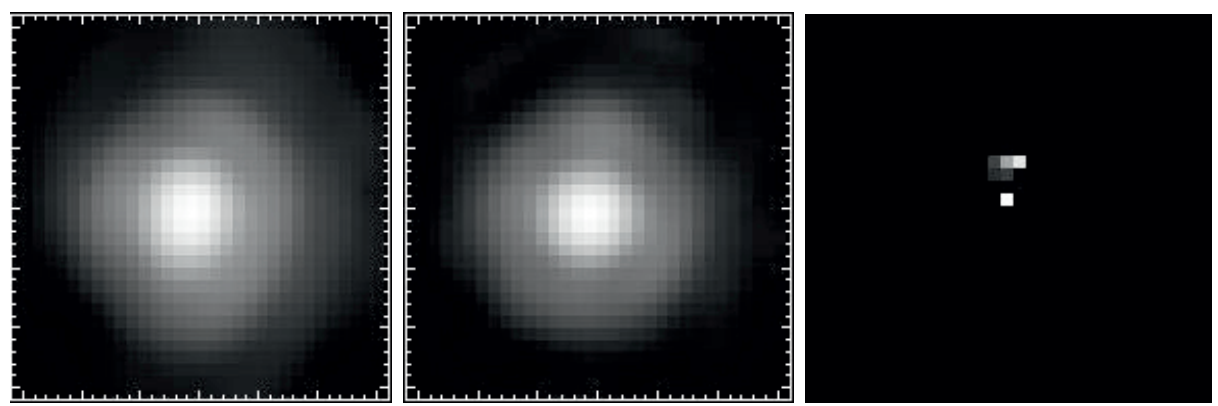

Fig. 10. From left to right: the observed image, the observed PSF, both pre-processed, and the result of a Lucy-Richardson-based super-resolution algorithm on the image.

\subsubsection{Going further in angular resolution}

Figure 10 shows a very preliminary (and unpublished) result of the application of a Lucy-Ricardson-based super-resolution algorithm (first evoked in Correia et al. 2002 and then described in details in Anconelli et al. 2005) on NACO/VLT data of the unresolved binary star HD 87643. It clearly unveils a very close binary star (separation smaller than half an element of resolution $\lambda / D$ ), with some possible matter around the component above, confirming previous AMBER/VLTI observations (Millour et al. 2009).

Thanks are due to Armando Riccardi and Olivier Lardière for having kindly provided both of them one of the illustrations presented for this course/paper. Thanks are also due to the organizers of the summer school for which this course/paper was prepared: Céline Theys-Ferrari, David Mary, and Claude Aime.

\section{References}

Aime, C., Borgnino, J., Martin, F., et al., 1986, J. Opt. Soc. Am. A, 3, 1001

Aime, C., 1987, J. Optics (Paris), 10, 318

Anconelli, B., Bertero, M., Boccacci, P., \& Carbillet, M., 2005, A\&A, 431, 747

Aristidi, É., Carbillet, M., Lyon, J.-F., \& Aime, C., 1997, A\&AS, 125, 139

Aristidi, É, Fossat, É., Agabi, A., et al., 2009, A\&A, 499, 955

Baldwin, J.E., Tubbs, R.N., Mackay, C.D., et al., 2001, A\&A, 368, L1

Brusa, G., Riccardi, A., Accardo, M., et al., 1999, in Proceedings of the Backaskog workshop on Extremely Large Telescopes, ed. T. Andersen, A. Ardeberg \& R. Gilmozzi, ESO Proc., 57, 18

Campbell, H.I. \& Greenaway, A.H., 2006, EAS Publication Series, 22, 165

Carbillet, M., 1996, Ph.D. Thesis (Université de Nice-Sophia Antipolis (UNS))

Carbillet, M., Aime, C., Aristidi, É., \& Ricort, G., 1998, A\&AS, 127, 569

Carbillet, M., Correia, S., Boccacci, P., \& Bertero, M., 2002, A\&A, 387, 743

Carbillet, M., Vérinaud, C., Esposito, S., et al., 2003, SPIE Proc., 4839, 131 
Carbillet, M., Vérinaud, C., Femenía Castellá, et al., 2005, MNRAS, 356, 1263

Carbillet, M., 2006, EAS Publication Series, 22, 121

Carbillet, M., Maire, A.-L., Le Roux, B., et al., 2010, EAS Publications Series, 40, 157

Carbillet, M., \& Riccardi, A., 2010, Appl. Opt., 49, JOSA A/App. Opt. Joint Feature Issue on Adaptive Optics, G167-G173

Carbillet, M., Riccardi, A., \& Xompero, M., 2012, SPIE Proc., 8447, 844762

Correia, S., Carbillet, M., Boccacci, P., Bertero, M., \& Fini, L., 2002, A\&A, 387, 733

Desiderà, G., \& Carbillet, M., 2009, A\&A, 507, 1759

Esposito, S., \& Riccardi, A., 2001, A\&A, 369, L9

Esposito, S., Riccardi, A., Fini, L., et al., 2010, SPIE Proc., 7736, 773609

Folcher, J.-P., 2013, EAS Publication Series, 59, 93

Giordano, C., Vernin, J., Chadid, M., et al., 2012, PASP, 124, 494

Labeyrie, A., 1970, A\&A, 6, 85

Labeyrie, A., \& Foy, R., 1995, A\&A, 152, L29

Lardière, O., Carbillet, M., Riccardi, A., et al., 2005, EAS Publication Series, 14, 291

Le Roux, B., Coyne, J., \& Ragazzoni, R., 2005, Appl. Opt., 44, 171

Mackay, C.D., Rebolo-López, R., Femenía Castellá, B., et al., 2012, SPIE Proc., 8446, 844672

Millour, F., Chesneau, O., Borges Fernandez, M., et al., 2009, A\&A, 507, 317

Nicolle, M., Fusco, T., Rousset, G., \& Michau, V., 2004, Opt. Lett., 29, 2743

Poyneer, R., \& Machintosh, J., 2004, J. Opt. Soc. Am. A, 350, L23

Ragazzoni, R., \& Farinato, J., 1999, A\&A, 350, L23

Riccardi, A., 2003, in Adaptive Optics Mini-School (Garching-bei-Muenchen, Germany, 19-21 Feb. 2003)

Riccardi, A., Xompero, M., Briguglio, R., et al., 2010, SPIE Proc., 7736, 77362C

Rigaut, F., \& Gendron, É., 1992, A\&A, 261, 677

Roddier, F., 1981, Progress in Optics XIX, 281, ed. E. Wolf (North-Holland, Pub. Co.)

Sarrazin, M., 1996, ESO Proc. on Topical Meeting on Adaptive Optics

Strehl, K., 1902, Zeit. Instrumenkde, 22, 213

Vérinaud, C., Le Louarn, M., Korkiakoski, V., \& Carbillet, M., 2005, MNRAS, 357, L26

Weigelt, G., 1977, Opt. Commun., 21, 55

Worden, S.P., Lynds, C.R., \& Harvey, J.W., 1976, J. Opt. Soc. Am., 66, 1243 